\title{
Correction: The medial tibial stress syndrome score: a new patient-reported outcome measure
}

Winters M, Moen MH, Zimmermann, WO, et al. The medial tibial stress syndrome score: a new patient-reported outcome measure. Br J Sports Med 2016;50:1192-9. doi: 10.1136/ bjsports-2015-095060.

This article was previously published with the following errors: ${ }^{1}$

1. The abstract (page 1) states that the intraclass correlation coefficient (ICC) for the medial tibial stress syndrome (MTSS) score was 0.81 .

2. The authors reported the MTSS score's standard error of measurement (SEM) to be 1.73 (page 6). The abstract (page 1) and Results section (page 6) state that the smallest detectable change for groups is 0.69 and for individuals 4.80 .

These statistics were incorrect:

1. The ICC for the MTSS score was 0.82 , as was correctly stated in the main text on page 6 .

2. The authors made an error calculating the SEM. The equation provided was $S E M=S D$ measurement $1+2 \sqrt{ } 1-$ ICC, where $S D=S D$ deviation. Here, the authors added the SDs of measurement 1 and 2 and used these consequently for calculating the smallest detectable change on the group and individual level. Adding the SD's of measurement 1 and 2 was incorrect and led to a spuriously large SEM, also affecting the smallest detectable change for groups and individuals.

The authors re-calculated the SEM, using current best practice to include random error only. ${ }^{2}$ Here, $\mathrm{SEM}$ is determined as $\mathrm{SEM}=\sqrt{ } \mathrm{MSe}$ where $\mathrm{MSe}$ is the mean square error from a repeated measures ANOVA. MTSS score's MSe $=0.758$. Consequently, $\mathrm{SEM}=\sqrt{ } 0.758=0.87$.

Following this SEM, the smallest detectable change (SDC) for groups and individuals are:

Groups:

$\mathrm{SDC}$ group $=\mathrm{SEM} * 1.96 * \sqrt{2} / \sqrt{ } \mathrm{n}=0.87 * 1.96 *(1.41 / 6.93)=0.35$

Individuals:

SDC_individual $=S E M * 1.96 * \sqrt{ } 2=0.87 * 1.96 * 1.41=2.41$

The interpretation on page 7 , that "this instrument may be limited for monitoring individual patients with MTSS" should be reconsidered following these calculations. The MTSS score may be able to detect individual changes beyond measurement error with $95 \%$ certainty as small as 2.41 points, and 0.35 for groups. The new calculations underpin the MTSS score's reliability and potential use for measuring changes in individual patients.

Supplemental online material one provides the MTSS score in Dutch and English with these accurate statistics.

(C) Author(s) (or their employer(s)) 2020. No commercial re-use. See rights and permissions. Published by BMJ. Br J Sports Med 2020;54:e2. doi:10.1136/bjsports-2015-095060corr1

\section{A Check for updates}

\section{REFERENCES}

1 Winters M, Moen MH, Zimmermann WO, et al. The medial tibial stress syndrome score: a new patient-reported outcome measure. Br J Sports Med 2016;50:1192-9.

2 Hopkins WG. Measures of reliability in sports medicine and science. Sports Med 2000;30:375-81. 